\title{
Index of places
}

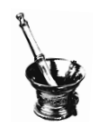

page numbers in italics refer to illustrations

The Americas i6, pl. 3

Maryland i93n.

Richmond, Virginia I72

Bath 40, I78n., I79n., 266n.

Bedfordshire

Carlton I, 6

Bristol I36n., 266

St Vincent's Spring 2I6, 266

Buckinghamshire $163 n$.

Aylesbury I54n.

Cambridge 6, 29, 31, 46, 67, 20 In.

University 29, 203n., 2I4n., 243 .

King's College 6

Queens' College I-6, 3I, 42-4, 46, $238 n$.

St Catherine's College 225 n.

Derby 208n.

Devon 99n.

Durham

Sledwich I8In.
Essex I27n.

France 2I, 3I, 33, 250

Douai 212

Montpellier 43

Paris 43

Germany 78

Frankfurt 46

Gloucester 178

Gloucestershire pl. 2

Aston-sub-Edge 225n.

Quinton 89, I32

Quorndon I45n.

Saintbury I86n.

Southam 87, iा6, I68n., I78, $245 \mathrm{n}$.

Stanton I86n.

Toddington I86

Hampshire

Combe 2ign.

Italy $25^{\circ}$

Padua 43

Tuscany pl. 23

Venice pl. 2I 


\section{Index of places}

Leicester 6, I45

Leicestershire in8n., pl. 2

Elmesthorpe 2I8n.

London I-3, 7-IO, I6-I8, 46-7, 58, 8on., 93n., II2, I28n., I54,

Blackfriars 3 216, 222n., 275n., pl. 3

British Library and Museum I8, 50, 54

College of Physicians I-3, I8, 32, 284

Little Britain 54

Middle Temple i22n., I49n.

St Gregory by St Paul's 38

St Paul's Cathedral $3^{8}$

Southwark, see Surrey

Westminster Abbey 95n.

Low Countries 62, I67, pl. 3

Delft pl. 22

Ludlow (and Ludlow Castle) 78, 8o-3, 86, 95n., 246n., pl. 2

Middlesex

Acton 7

Norfolk 26in.

Northamptonshire in In.

Abington IIIn.

Castle Ashby 8on.

Hardingstone 2I4n., 2I5

Northampton 59, 2I 4

Norwich 9-IO

Nottinghamshire I26n.

Oxford 47, 66, 21 I, 222n., 245n., $266 n$.

University 83n., I62n., I74n., 2IIn., 236n., 243n., 25In., $277 n$.

Balliol College ir5n., I33n., $236 n$.

Bodleian Library 47

Magdalen College i85n.

Queen's College 245n.
Oxfordshire I87n., pl. 2

Banbury I9

Broughton Castle 258n.

Burford 195, 244n.

Charlbury igon.

Walcot igon.

Shrewsbury I67

Stratford-upon-Avon I-27, 30, 44-7, 57, 81, 83, 85, 87, 92n., 93n., 96, IOI, I03-4n., II5n., I3on., I35, I38, I4I, I48n., I50, I52n., I54, I62, I65n., I8In., I83n., ı9I, I95n., I96-8, 203, 2I5, 2I6, 220n., 226, 230, 234-5, 240n., 244n., 266n., 277n., pl. 2

Bear Inn 85, I03-4n., I65n.

Bridge Street 85n., I03-4n., I65n.

Clopton House i8In.

Ecclesiastical Court 4

Gospel Bush 8-9

Hall's Croft 6-7, 7

Holy Trinity Church 2, 6, I2-I4, I4, 25-6, 26, i15n., I36n., I52n., 252n.

King's New School 277n., 279

New Place 2, 6, 8, 93n., I36n., $255^{\mathrm{n}}$.

St Mary's i36n.

Stratford Old Town 6, 7, I52, 200 .

Welcombe 8, I97n.

Surrey

Mitcham 92n.

Southwark 78

Sussex I7In., I87n., $242 n$.

Ladyholt i7in.

Wales 8o, 82, I49, I52, 246

Glamorgan 236n.

Warwick I8-I9, 54, I22, I4In., I47n., I95n., 206n., 242n., 243n., 245n., 266n., 268

Warwick Castle 257 


\section{Index of places}

Warwick Priory I7In., I72, I95n., 206n., 242n.

Warwickshire 95, I33, I85n., 206n., 242n., 266n., pl. 2

Alcester 89n., i30, I48n.

Alveston 92, I92, I93

Arrow I2In., 204n

Aston Cantlow i37n.

Avon Dassett 232.

Barlichway Hundred i64n.

Bidford-on-Avon (and Bidford Grange) I64., 208n.

Billesley igon.

Binton 23on.

Birdingbury 245n.

Birmingham 240n.

Bishops Tachbrook 2i6n.

Brailes I32n.

Broom Court 20In., 208

Budbrooke I47n.

Bushwood 24-5

Cawston 97n. 98

Cherington 212n.

Clifford Chambers i9, 95n., 96, I49n., I50, I5In., I56, 2 I9n., 250n, $25 I$

Compton Wynyates $222 n$.

Coughton (and Coughton Court) i $18 n .$, i66n.

Dunchurch 97n.

Emscote i40n., I43n., I53n.

Ettington I74n., I84n., I85n., $202 n$.

Hampton Lucy 6

Hartshill 96

Honington 120, I25n., I3On.

Idlicote $255 \mathrm{n}$.

Ilmington I IO, I8on.

King's Coughton 247

Knowle I2 I

Lambcote 202

Lawford I39

'Libington' [Luddington?] 92

Long Marston izon.

Loxley 202n., 234n., 275
Milcote I6I

Moreton Morrell I33n., I58n., I74n., I75

Newbold-on-Avon i39n.

Newnham 137

Norton Curlieu 147

Oversley I2In., I3In.

Oxhill I32n., I74n., 202n.

Packwood Hall 232n.

Pillerton Priors 158.

Polesworth 25I

Radford Semele i66n., I72, 248

Ragley 204n.

Rowington I63n.

Salford Priors I3on., i63n., 208n.

Shipston I07

Snitterfield 26gn.

Stratford-upon-Avon, see separate entry

Tachbrook Mallory 2i6n.

Talton I28n. I3on., I49n.

Temple Grafton I3I

Tredington I3On., I5In.

Walton i i8n.

Warwick, see separate entry

Weston I27n., 212, 277n.

Weston House 127n., I29, 212n.

Whitnash 245n.

Wood Bevington 97n., I33n., I63n.

Wootton Wawen i87n.

Worcester I3, IOI, I24, I4In., I55, I6I, pl. 2

Worcester Cathedral 252n., 253

Worcestershire 3, 9In., I40, I45, 247n., 266n., pl. 2

Bell-End I26

Bengeworth 270

Beoley I26n.

Bromsgrove i3In., 263n.

Cook Hill iorn., 26r

Cookhill Priory 26in.

Evesham 7

Feckenham ${ }_{4} 6$

Flyford Favell gon. 


\section{Index of places}

Worcestershire (cont.)

Grafton I3In., I67n.

Hanbury 236

Harvington 238n.

Huddington i i7n., I88n.

Huddington Court 188

Inkberrow 9In., IoIn., I8on.

Lindridge $\mathrm{I} 6 \mathrm{In}$.
Morton Underhill gon.

Ombersley i i4n., I8o

Pebworth I2I

Rous Lench 145

Stock Green 90

Westwood Park I4In., I42, pl. 2

White Ladies Aston I24

Worcester, see separate entry 